\title{
Linear Kinetic Heat Transfer: Moment Equations, Boundary Conditions, and Knudsen layers
}

\author{
H. Struchtrup* \\ ETH Zürich, Department of Materials, Polymer Physics, CH-8093 Zürich, Switzerland
}

Physica A, doi:10.1016/j.physa.2007.11.044

\begin{abstract}
A linear kinetic equation for heat transfer is solved by means of the method of moments. The moment equations are solved with Maxwelltype boundary conditions for steady state energy transport. The results exhibit marked Knudsen boundary layers. The accuracy of the description is examined, and it is shown that already a relatively small number of moments can give satisfactory resolution of Knudsen layers for Knudsen numbers $\varepsilon \leq 1$. The implications for moment equations for more complicated kinetic equations (such as the Boltzmann equation) are discussed.
\end{abstract}

\section{Introduction}

The ongoing miniaturization of technical devices requires accurate theories for simulations of flows on the microscale [1]. Microscale gas flows are described by kinetic theory, where linear effects are dominant [2]. This paper focusses on a particularly important feature of such flows, the modelling of Knudsen layers.

Kinetic theory describes transport processes in gases resulting from the interplay of free flight and interaction of the gas particles among themselves and/or with other objects. Mathematically the gas is described by a particle distribution function which follows as the solution of a kinetic equation. Well-known kinetic equations are the Boltzmann equation for classical gases [3][4], its variants for electrons [5] and phonons [6], and the radiative transfer equation [7].

The solution of any kinetic equation is usually cumbersome, and numerically expensive, and thus one branch of kinetic theory derives reduced systems, socalled macroscopic models, from the kinetic equations by various means. The classical method to this end is the Chapman-Enskog method which expands the distribution function into a series in a smallness parameter, typically the ratio between the mean free path an the desired resolution, the Knudsen number [3][8]. In many cases the Chapman-Enskog expansions to higher order lead to unstable equations [9][10] or unphysical results [11].

\footnotetext{
${ }^{*}$ On leave from: Dept. of Mechanical Engineering, University of Victoria, Victoria BC, Canada, struchtr@uvic.ca
} 
An alternative that avoids instabilities is provided by Grad's method of moments [12][13] in which the kinetic equation is replaced by a finite set of equations for the moments of the distribution function. The equations are obtained by taking weighted averages of the kinetic equation over the microscopic variable. The phase density is expressed through the moments by means of a series expansion into orthogonal functions, and a truncation of the series yields the required closure conditions. The moment method and its variants were applied to ideal gases [12]-[23], electrons in semiconductors [24][25], radiative transfer [7][26], and phonon transport in crystals [6].

Despite the long history, and success, of the moment method, there are still unresolved issues and open questions. The most important problems are to design boundary conditions for the moment equations, and to find useful criteria for the number of moment equations that should be taken into account for particular problems.

The question of boundary conditions was tackled recently in [22] and [23], where it was shown that the boundary conditions for the kinetic equation can be used to derive meaningful boundary conditions for moment equations. In the case of non-linear equations additional boundary conditions are obtained from the bulk equations [23].

Combination of the Chapman-Enskog and Grad methods [18][19][20] leads to the regularized 13 moment equations which are of third order in the Knudsen number (super-Burnett order) in the Chapman-Enskog sense. The ChapmanEnskog method, and the methods employed in [18][19][20], are based solely on the transport equations in the bulk, and do not consider boundary effects or boundary conditions. Thus, the Chapman-Enskog order of accuracy is only valid in the bulk, but not necessarily adjacent to walls or interfaces, where Knudsen boundary layers appear [4][27].

Knudsen layers are essentially linear contributions, and thus can be computed from the linearized equations. In strongly non-linear flows, non-linear bulk effects [21][8] are more important than Knudsen layers, even close to the walls. While the magnitude of the non-linear bulk contributions is related to the Knudsen number through the Chapman-Enskog method, the discussion of the Knudsen layers must include the boundary conditions, which, after all, determine their amplitudes.

The main aim of this paper is to better understand the behavior of Knudsen layers within the realm of moment equations. It was shown earlier that in Gradtype moment equations the moments contain superpositions of a multitude of Knudsen layers of different widths [17]; the amplitudes of the Knudsen layers, however, were not discussed. Thus, the aim of the present paper is to discuss the amplitudes, and relevance, of the Knudsen layers.

To simplify the discussion, we consider a linear kinetic model for one-dimensional energy transfer, which allows analytical computations for arbitrary number of moments. The number of Knudsen layers grows with the number of moments considered. The results indicate that a relatively low number of moments suffices to give results of reasonable accuracy as long as Knudsen layers with a variety of widths are present.

The remainder of the paper is organized as follows: Section 2 introduces the simple kinetic model for heat transfer which is similar to the linearized phonon-Boltzmann equation, and the corresponding Maxwell-type boundary conditions. The moments, their equations and boundary conditions are derived 
from the kinetic model in Section 3. Section 4 discusses asymptotic methods and approximations for the bulk. The steady state heat transfer problem is solved in Section 5 and it is shown that all moments are superpositions of Knudsen layers of various widths. Asymptotics for the Knudsen layers based on smallness of the Knudsen number shows that Knudsen layers can be ignored in a first order theory, but not when higher order in the Knudsen number is desired. Jump boundary conditions for energy arise from the asymptotics quite naturally, and the Knudsen layer correction of the jump condition is discussed. Results for a variety of Knudsen numbers and moment numbers are compared in Section 6 . The paper closes with some conjectures on the behavior of moment equations for more complicated, and more realistic, moment equations.

\section{The kinetic model and its properties}

\section{$2.1 \quad$ Kinetic model}

We consider a simple transport equation for one-dimensional heat transfer in dimensionless form. The energy transfer is effected by particles that travel with unit velocity in three dimensional space, and carry energy. Thus, on the microscopic level the particles are described through their location in spacetime $(x, t)$, and their direction which is expressed through the direction cosine $\cos \vartheta=\mu \in[-1,1]$, where $\vartheta$ is the angle between the particle direction and the $x$-axis. The particle distribution function $f(x, t, \mu)$ is defined such that $f d x d \mu$ gives the energy of particles in the interval $(x, x+d x)$ with direction cosines in $(\mu, \mu+d \mu)$ at time $t$. Thus, the energy density is

$$
\lambda_{0}(x, t)=\int_{-1}^{1} f(x, t, \mu) d \mu .
$$

It is assumed that the particles undergo collisions (among each other, or with a background medium) which only conserve energy; the dimensionless mean free path, i.e. the Knudsen number, is denoted by $\varepsilon$.

The kinetic equation for this simple transport model reads

$$
\frac{\partial f}{\partial t}+\mu \frac{\partial f}{\partial x}=-\frac{1}{\varepsilon}\left(f-f_{E}\right)
$$

where $f_{E}=f_{E}(x, t)$ is the isotropic equilibrium distribution function. The right hand side of the above equation is a BGK-type collision term that describes isotropic scattering [7].

Integration of (2) over all directions gives the balance of energy as

$$
\frac{\partial \lambda_{0}}{\partial t}+\frac{\partial \lambda_{1}}{\partial x}=0,
$$

where the energy flux is defined as

$$
\lambda_{1}=\int_{-1}^{1} \mu f(x, t, \mu) d \mu .
$$

Conservation of energy requires that the integral of the collision term vanishes, so that

$$
\int_{-1}^{1} f(x, t, \mu) d \mu=\int_{-1}^{1} f_{E}(x, t) d \mu .
$$


From this relation we can identify the equilibrium distribution as

$$
f_{E}(x, t)=\frac{1}{2} \lambda_{0}(x, t) .
$$

\subsection{Boundary conditions}

The kinetic equation must be furnished with boundary conditions in order to formulate meaningful problems. We adopt a variant of Maxwell's accommodation model [28], which states that a fraction $\chi$ of the particles impinging on a wall are thermalized, and leave in an equilibrium function defined by the properties of the wall, while the remaining fraction $(1-\chi)$ of particles is specularly reflected. The 1-dimensional normal $\gamma$ is defined such $\gamma=1$ at the left wall $(x=-1 / 2)$ and $\gamma=-1$ at the right wall $(x=1 / 2)$, and the distribution function at the walls reads

$$
\bar{f}=\left\{\begin{array}{cc}
\chi f_{W}+(1-\chi) f(-\gamma \mu) & , \gamma \mu>0 \\
f(\gamma \mu) & , \gamma \mu<0
\end{array} .\right.
$$

Here, $f_{W}=\frac{1}{2} \lambda_{W}$ denotes the equilibrium distribution at wall conditions.

\subsection{Similarity to Phonon model}

In the above we abstained from assigning a concrete physical picture to the model. Altogether one might think of a, simplified, model for heat transfer in a pure crystal, described through phonon transport [6]. In our simplified model phonon frequency is ignored, which can be done under the assumption that the collision frequency is independent of phonon frequency, since this allows integration over frequency. Typically phonons undergo interactions with themselves and impurities in the lattice, and the simplified model only considers what is known as R-processes, where energy is conserved, and ignores the so-called N-processes, which describe phonon-phonon interactions where energy and momentum are conserved. The "walls" of the boundary conditions are interfaces between a pure material (with relatively large $\varepsilon$ ), and a less pure material (with small $\varepsilon$ ). Moreover, we refrain from defining temperature, and will use solely energy densities $\lambda_{0}$ and $\lambda_{W}$, which are, however, measures for temperature. In particular thermal equilibrium implies $\lambda_{0}=\lambda_{W}$, which corresponds to the zeroth law of thermodynamics. Similar transport equations appear in radiative transfer [7] and neutron transport [29].

\section{Moments, their equations and boundary con- ditions}

\subsection{Moment equations}

The moment method provides a means to solve the kinetic equation approximately. The quality of the approximate solution is the main concern of this paper. For the simple problem at hand, moments based on Legendre polynomials are the obvious choice [7][29]. Legendre polynomials form an orthogonal 
set by means of the relation $\int_{-1}^{1} P_{n} P_{m} d \mu=\frac{2}{2 n+1} \delta_{n m}$, moreover they obey the recurrence relation $\mu P_{n}(\mu)=\frac{n}{2 n+1} P_{n-1}(\mu)+\frac{n+1}{2 n+1} P_{n+1}(\mu)$. We define the $P_{n}$-moments of $f$ as

$$
\lambda_{n}=\int_{-1}^{1} P_{n}(\mu) f d \mu .
$$

For the desired degree of approximation, we consider a finite number of moments, $\lambda_{n}(n=0,1, \ldots, N)$. The first two moments are energy density and energy flux.

Due to the orthogonality of the Legendre polynomials, the distribution function can be expressed through its moments as

$$
f^{(N)}=\sum_{n=0}^{N}\left(n+\frac{1}{2}\right) P_{n}(\mu) \lambda_{n} .
$$

Plugging this into the kinetic equation (2) and use of the recurrence relation yields

$$
\begin{gathered}
\sum_{n=0}^{N-1}\left(n+\frac{1}{2}\right) P_{n}(\mu)\left[\frac{\partial \lambda_{n}}{\partial t}+\frac{n}{2 n+1} \frac{\partial \lambda_{n-1}}{\partial x}+\frac{n+1}{2 n+1} \frac{\partial \lambda_{n+1}}{\partial x}+\frac{1}{\varepsilon}\left(\lambda_{n}-\lambda_{0} \delta_{n 0}\right)\right] \\
+\frac{2 N+1}{2} P_{N}(\mu)\left[\frac{\partial \lambda_{N}}{\partial t}+\frac{N}{2 N+1} \frac{\partial \lambda_{N-1}}{\partial x}+\frac{1}{\varepsilon}\left(\lambda_{N}-\lambda_{0} \delta_{N 0}\right)\right] \\
+P_{N+1}(\mu) \frac{N+1}{2} \frac{\partial \lambda_{N}}{\partial x}=0
\end{gathered}
$$

Taking the $P_{n}$-moments of this expression for $n=0,1, \ldots, N$ yields a closed set of moment equations for the $\lambda_{n}(n=0,1, \ldots, N)$,

$$
\begin{aligned}
\frac{\partial \lambda_{0}}{\partial t}+\frac{\partial \lambda_{1}}{\partial x} & =0 \\
\frac{\partial \lambda_{n}}{\partial t}+\frac{n}{2 n+1} \frac{\partial \lambda_{n-1}}{\partial x}+\frac{n+1}{2 n+1} \frac{\partial \lambda_{n+1}}{\partial x} & =-\frac{1}{\varepsilon} \lambda_{n} \\
\frac{\partial \lambda_{N}}{\partial t}+\frac{N}{2 N+1} \frac{\partial \lambda_{N-1}}{\partial x} & =-\frac{1}{\varepsilon} \lambda_{N}
\end{aligned}
$$

The first equation $(8)_{1}$ is just the energy equation (3). For $N \rightarrow \infty$ the (infinite) set of moment equations is equivalent to the kinetic equation for the full distribution function $f=f^{(\infty)}$. For finite $N$, however, the truncated distribution function $f^{(N)}(6)$ and the finite moment system (8) are not fully equivalent to the kinetic equation since the last term in $(7), P_{N+1}(\mu) \frac{N+1}{2} \frac{\partial \lambda_{N}}{\partial x}$, does not vanish. This term is the sole remainder of the moment equation for $\lambda_{N+1}$ and occurs as a result of truncation, which implies ignoring all higher moments $\lambda_{N+\alpha}$ $(\alpha \geq 1)$. Thus, truncation at finite $N$ yields an error, and $N$ must be chosen large enough in order to make the error small.

\subsection{Boundary conditions}

The boundary conditions for the $\lambda_{n}$ follow by evaluating the moments with the phase density at the boundary (5), which gives (overbars indicate values at the 
boundaries)

$$
\bar{\lambda}_{n}=\chi \int_{\gamma \mu=0}^{1} P_{n}(\mu)\left[f_{W}-f(-\gamma \mu)\right] d \mu+\left[1+(-1)^{n}\right] \int_{\gamma \mu=0}^{1} P_{n}(\mu) f(-\gamma \mu) d \mu .
$$

where we used that Legendre polynomials $P_{n}(\mu)$ with even (odd) coefficients are even (odd) functions of $\mu$.

Equation (9) provides one boundary condition for every moment at each wall, which gives more boundary conditions than mathematically required. Grad showed that only boundary conditions for odd moments should be considered [12]. His argument is based on a special case with a specularly reflecting wall, where $\chi=0$, and where the distribution function is even, $f(\mu)=f(-\mu)$. For (9) applied to even moments this implies mere identities, $\bar{\lambda}_{n}=\bar{\lambda}_{n}$, which are meaningless as boundary conditions, and thus, in order to have continuity in $\chi$, (9) must be considered only for odd moments.

For the computation of the boundary conditions for odd moments we split the distribution function into an even and an odd part, so that $f(\mu)=f_{e}(\mu)+f_{o}(\mu)$ and $f(-\gamma \mu)=f_{e}(\mu)-\gamma f_{o}(\mu)$. Since odd moments are determined solely by $f_{o}$, evaluation of $(9)$ gives

$$
\bar{\lambda}_{n}=\gamma \frac{2 \chi}{2-\chi} \int_{\mu=0}^{1} P_{n}(\mu)\left[f_{W}-f_{e}(\mu)\right] d \mu \quad(n \text { odd }) .
$$

Since only even Legendre polynomials contribute to $f_{e}(\mu)$, we obtain the boundary conditions from (6) as

$$
\bar{\lambda}_{n}=-\gamma \frac{2 \chi}{2-\chi}\left(\Psi_{n 0}\left[\bar{\lambda}_{0}-\lambda_{W}\right]+\sum_{m=2, m \text { even }}^{N} \Psi_{n m} \bar{\lambda}_{m}\right) \quad(n \text { odd }),
$$

where the coefficients are half-space integrals of Legendre polynomials,

$$
\Psi_{n m}=\left(m+\frac{1}{2}\right) \int_{0}^{1} P_{n}(\mu) P_{m}(\mu) d \mu .
$$

Numerical evaluation shows that, while all entries $\Psi_{n m}$ are different from zero, the largest entries are close to the diagonal, so that the boundary condition for the odd moment $\bar{\lambda}_{n}$ is mainly given through the values of the neighbouring even moments $\bar{\lambda}_{n-1}$ and $\bar{\lambda}_{n+1}$.

\section{Asymptotics and Approximations}

\subsection{Chapman-Enskog expansion}

In the next two sub-sections we examine the reduction of the moment system (8) by asymptotic methods based on the Knudsen number $\varepsilon$ as smallness parameter.

First we consider the Chapman-Enskog (CE) expansion [3] [4] of the moment equations [8], which we apply to those moments that are not conserved (i.e. all but the energy $\lambda_{0}$ ). For the CE expansion, the moments are written as a power series in $\varepsilon$

$$
\lambda_{n}=\sum_{\alpha=0} \varepsilon^{\alpha} \lambda_{n}^{(\alpha)} \quad(n \geq 1)
$$


The expansion is inserted into the moment equations, and contributions at the various powers of $\varepsilon$ are equated.

Since the production terms on the right hand side are the only contributions with power $\varepsilon^{-1}$, the zeroth order contributions must vanish,

$$
\lambda_{n}^{(0)}=0 \quad(n \geq 1) .
$$

Evaluation of the equation for the energy flux $\lambda_{1}$ to leading order gives Fourier's law,

$$
\lambda_{1}^{(1)}=-\frac{1}{3} \frac{\partial \lambda_{0}}{\partial x} .
$$

The last two equations $(13,14)$ imply that the energy flux is of first order in $\varepsilon$, since its first order contribution $\lambda_{1}^{(1)}$ does not vanish. Thus, in the energy equation $(8)_{1}$ the second term is of order $\varepsilon$, and, since the equation has only two terms, the first term must be of order $\varepsilon$ as well. Since the energy density $\lambda_{0}$, which is an equilibrium quantity, remains unscaled, we have to conclude that the time derivative $\partial / \partial t$ must be rescaled to $\varepsilon \partial / \partial \tilde{t}$, that is, within the $\mathrm{CE}$ expansion, we have to apply diffusion scaling.

With the diffusion scaling we obtain with the ansatz (12) the expansion coefficents at zeroth order (13). The first order coefficents are

$$
\lambda_{1}^{(1)}=-\frac{1}{3} \frac{\partial \lambda_{0}}{\partial x} \quad, \quad \lambda_{n}^{(1)}=0(n \geq 2) .
$$

Thus, only the first moment, $\lambda_{1}$, has a first order contribution, while all other moments are at least of second order.

For the second order we find

$$
\lambda_{1}^{(2)}=0 \quad, \quad \lambda_{2}^{(2)}=\frac{2}{15} \frac{\partial^{2} \lambda_{0}}{\partial x^{2}} \quad, \quad \lambda_{n}^{(2)}=0(n \geq 3),
$$

and for higher orders

$$
\begin{aligned}
& \lambda_{1}^{(\alpha+1)}=-\frac{\partial \lambda_{1}^{(\alpha-1)}}{\partial \tilde{t}}-\frac{2}{3} \frac{\partial \lambda_{2}^{(\alpha)}}{\partial x}, \\
& \lambda_{n}^{(\alpha+1)}=-\frac{\partial \lambda_{n}^{(\alpha-1)}}{\partial \tilde{t}}-\frac{n}{2 n+1} \frac{\partial \lambda_{n-1}^{(\alpha)}}{\partial x}-\frac{n+1}{2 n+1} \frac{\partial \lambda_{n+1}^{(\alpha)}}{\partial x} \quad(n \geq 2) .
\end{aligned}
$$

It is rather straightforward to conclude that

$$
\lambda_{n}^{(\alpha-1)}=0 \text { for } \alpha \leq n .
$$

In other words, for the moment $\lambda_{n}$ the first non-vanishing coefficient is $\lambda_{n}^{(n)}$ and we can say that the $n$-th moment is of order $\mathcal{O}\left(\varepsilon^{n}\right)$. Space restrictions forbid to further discuss higher order results from the Chapman-Enskog method, which can be unstable, or lead to unphysical behavior [9][10][8].

\subsection{Order of Magnitude Method}

An alternative expansion is offered by the order of magnitude method, which uses the results from the Chapman-Enskog expansion as a measure for the expected magnitude of the moments, but is not interested in the actual expansion (12). The method proceeds in three steps as outlined below [20][8]. 
The first step is the determination of the order of magnitude of the moments by means of the $\mathrm{CE}$ expansion as in the previous section. We can make the result (15) more explicit by writing

$$
\lambda_{n}=\varepsilon^{n} \tilde{\lambda}_{n}
$$

where the rescaled moments $\tilde{\lambda}_{n}$ are of order unity, while the magnitude is given by the powers of $\varepsilon$.

The second step consists of combining the variables such that at any order in $\varepsilon$ there is a minimum number of variables [20][8]. Since, by (15), we have only the moment $\lambda_{n}$ with order of magnitude $\mathcal{O}\left(\varepsilon^{n}\right)$, no further reduction is possible, and this step is not required anymore.

The third step consists in reducing the infinite set of moment equations by considering the order of magnitude one wishes to achieve in the conservation law for energy. We perform the first few steps, for the case of diffusion scaling: The first three moment equations in the rescaled variables read

$$
\begin{gathered}
\varepsilon \frac{\partial \lambda_{0}}{\partial \tilde{t}}+\varepsilon \frac{\partial \tilde{\lambda}_{1}}{\partial x}=0, \\
\varepsilon^{2} \frac{\partial \tilde{\lambda}_{1}}{\partial \tilde{t}}+\frac{1}{3} \frac{\partial \lambda_{0}}{\partial x}+\varepsilon^{2} \frac{2}{3} \frac{\partial \tilde{\lambda}_{2}}{\partial x}=-\tilde{\lambda}_{1}, \\
\varepsilon^{3} \frac{\partial \tilde{\lambda}_{2}}{\partial \tilde{t}}+\varepsilon \frac{2}{5} \frac{\partial \tilde{\lambda}_{1}}{\partial x}+\varepsilon^{3} \frac{3}{5} \frac{\partial \tilde{\lambda}_{3}}{\partial x}=-\varepsilon \tilde{\lambda}_{2} .
\end{gathered}
$$

The equations to first order in $\varepsilon$ are obtained by considering the energy balance and only the leading term of the balance of the energy flux, $\tilde{\lambda}_{1}$, which is, again, Fourier's law $\frac{1}{3} \frac{\partial \lambda_{0}}{\partial x}=-\tilde{\lambda}_{1}$.

The second order equations result by adding the contribution of order $\varepsilon^{1}$ in the balance for $\tilde{\lambda}_{1}$, which is absent. Thus, the second order agrees with the first order.

The third order equations are obtained by adding the next higher contribution in $\varepsilon$ which is the full equation for $\tilde{\lambda}_{1}$. In this equation $\tilde{\lambda}_{2}$ appears, and is required to its leading order from the equation for $\tilde{\lambda}_{2}$ which gives (after division by $\varepsilon$ )

$$
\frac{2}{5} \frac{\partial \tilde{\lambda}_{1}}{\partial x}=-\tilde{\lambda}_{2} .
$$

Since the next contribution to $\tilde{\lambda}_{2}$ is two orders higher than the leading order, the fourth order agrees with the third order equations. For the fifth order, the full balance for $\tilde{\lambda}_{2}$ must be considered together with the leading contribution of the equation for $\tilde{\lambda}_{3}$. This is also the sixth order. And so on.

Thus, the following picture emerges: When diffusion scaling is employed in the order of magnitude method for the moment equations (8), the equations of order $\mathcal{O}\left(\varepsilon^{2 N}\right)$ (which agree with those of order $\mathcal{O}\left(\varepsilon^{2 N-1}\right)$ ) are given by the truncated set

$$
\begin{aligned}
\frac{\partial \lambda_{0}}{\partial t}+\frac{\partial \lambda_{1}}{\partial x} & =0 \\
\frac{\partial \lambda_{n}}{\partial t}+\frac{n}{2 n+1} \frac{\partial \lambda_{n-1}}{\partial x}+\frac{n+1}{2 n+1} \frac{\partial \lambda_{n+1}}{\partial x} & =-\frac{1}{\varepsilon} \lambda_{n}(n=1, \ldots, N-1), \\
\frac{N}{2 N+1} \frac{\partial \lambda_{N-1}}{\partial x} & =-\frac{1}{\varepsilon} \lambda_{N} .
\end{aligned}
$$


The only difference to the set (8) is the absence of the time derivative $\frac{\partial \lambda_{N}}{\partial t}$ in the last equation, which is a consequence of the diffusion scaling. Without diffusion scaling we find by the same set of arguments that the equations (17) are of order $\mathcal{O}\left(\varepsilon^{2 N-1}\right)$, while the set (8) turns out to be of order $\mathcal{O}\left(\varepsilon^{2 N}\right)$.

\section{$5 \quad$ Knudsen layers in steady state energy transfer}

\subsection{Knudsen layer solutions}

Due to the strong anisotropy of the distribution function at the boundary which is inflicted by the boundary condition (5) there appear transition layers near an interface, the so called Knudsen layers. In this section we shall compute and discuss the Knudsen layers for the moment system (8) in steady state processes, where the equations read

$$
\begin{aligned}
\frac{d \lambda_{1}}{d x} & =0 \\
\frac{1}{3} \frac{d \lambda_{0}}{d x}+\frac{2}{3} \frac{d \lambda_{2}}{d x} & =-\frac{1}{\varepsilon} \lambda_{1}, \\
\frac{n}{2 n+1} \frac{d \lambda_{n-1}}{d x}+\frac{n+1}{2 n+1} \frac{d \lambda_{n+1}}{d x} & =-\frac{1}{\varepsilon} \lambda_{n}, \\
\frac{N}{2 N+1} \frac{d \lambda_{N-1}}{d x} & =-\frac{1}{\varepsilon} \lambda_{N} .
\end{aligned}
$$

The corresponding boundary conditions are given by (10) at the left and right walls where

$$
\text { left: } x=-1 / 2, \gamma=1, \lambda_{L}^{W}=-1 \quad \text {, right: } x=1 / 2, \gamma=-1, \lambda_{R}^{W}=1 .
$$

Since the moment equations are linear, the values of $\lambda_{L, R}^{W}$ affect the amplitudes of all moments linearly, and thus it suffices to study the results for only one value. Non-symmetric values of $\lambda_{L, R}^{W}$ would shift the energy curve up or down.

From the first two equations (18) we obtain

$$
\lambda_{0}=K-\frac{3}{\varepsilon} \lambda_{1} x-2 \lambda_{2} \quad, \quad \lambda_{1}=\text { const. }
$$

where $K$ and $\lambda_{1}$ (the energy flux) are constants of integration. The energy $\lambda_{0}$ has a linear contribution, $K-\frac{3}{\varepsilon} \lambda_{1} x$, as is known from Fourier's law, plus an additional contribution, $-2 \lambda_{2}$, which describes the influence of higher moments.

The equations for the variables $\left\{\lambda_{2}, \lambda_{3}, \ldots, \lambda_{N}\right\}$ can be written in matrix form as

$\mathcal{B}_{\alpha \beta} \frac{\partial \lambda_{\beta}}{\partial x}=-\frac{1}{\varepsilon} \lambda_{\alpha}$ with $\mathcal{B}_{\alpha \beta}=\frac{\alpha+1}{2 \alpha+3} \delta_{\alpha, \beta-1}+\frac{\alpha+2}{2 \alpha+3} \delta_{\alpha, \beta+1}, \alpha, \beta=2, \ldots, N$

This system can be easily solved by diagonalization of the matrix $\mathcal{B}_{\alpha \beta}$. We denote the eigenvalues by $b^{(\alpha)}$, the matrix of eigenvectors by $\Phi_{\alpha \beta}$ and the diagonalized variables as $\Gamma_{\alpha}$ so that

$$
\lambda_{\alpha}=\sum_{\beta=2}^{N} \Phi_{\alpha \beta} \Gamma_{\beta} .
$$


Then, the system (21) assumes the form

$$
b^{(\alpha)} \frac{d \Gamma_{\alpha}}{d x}=-\frac{1}{\varepsilon} \Gamma_{\alpha}
$$

with the solution

$$
\Gamma_{\alpha}=\Gamma_{\alpha}^{0} \exp \left[-\frac{x}{\varepsilon b^{(\alpha)}}\right] .
$$

These are Knudsen layer solutions with amplitudes $\Gamma_{\alpha}^{0}$, and $\varepsilon b^{(\alpha)}$ is (a measure for) the width of the layers. From (22) we note that the moments $\left\{\lambda_{2}, \lambda_{3}, \ldots, \lambda_{N}\right\}$ are superpositions of Knudsen layers of different widths. For all $N$ the eigenvalues assume values in $[-1,1]$.

The solution of the boundary value problem follows from combining (20, $22,23)$ with the boundary conditions $(10,19)$ and solving for the constants of integration $K, \lambda_{1}, \Gamma_{\alpha}^{0}(\alpha=1, \ldots, N-1)$. This linear problem was solved by means of Mathematica ${ }^{\circledR}$.

The classical description of heat transfer is the case $N=1$, which has only energy as variable and the energy flux is given by Fourier's law (14). For $N=2$ the equations are the same as for $N=1$. Systems with even value of $N$ always have one zero eigenvalue, and $N-2$ non-zero eigenvalues, while systems with odd values have $N-1$ non-zero eigenvalues. All non-zero eigenvalues appear as pairs $\pm b$. The results show only little difference between a system with odd $N$, and the next higher system $N+1$, which has the same number of non-zero eigenvalues; therefore we shall present only curves for odd $N$.

A smaller accommodation factor only increases the energy jump, and lowers the amplitudes of the boundary layers, but does not affect the overall behavior of the solutions. Thus in all computations the accommodation factor is $\chi=1$.

\section{$5.2 \quad$ Scaling}

Equation (23) indicates that the proper space variable for resolving Knudsen layers is the stretched variable $X=x / \varepsilon$. When the stretched variable $X$ is used in the moment system, the parameter $\varepsilon$ vanishes from the equations. It follows that the asymptotic methods of Sec. 4 cannot be used to reduce the number of equations as soon as Knudsen layer effects become important [4].

Indeed, the expansions in Sec. 4 are relevant only for regions outside the Knudsen layers, i.e., in the bulk, since they are based solely on the transport equations. The influence of the Knudsen layers on the results, however, is due to the amplitudes $\Gamma_{\alpha}^{0}$, which are determined by the boundary conditions (10) in interplay with the moment equations.

The moments split into two groups: energy and energy flux (20), which have bulk and possibly Knudsen layer contributions, and the higher moments $\left\{\lambda_{2}, \lambda_{3}, \ldots, \lambda_{N}\right\}$, which consist only of Knudsen layer contributions (23).

We study the asymptotic behavior for steady state heat transfer for small $\varepsilon$. All moments are excited by non-zero values of $\left(\lambda_{R}^{W}-\lambda_{L}^{W}\right)$, and we ask for their magnitude at the boundary in terms of $\varepsilon$. For this, we consider the following list of arguments:

1. The only quantity controlled from the outside is the average energy gradient between the two walls (at distance $\Delta x=1$ ),

$$
\left\langle\frac{d \lambda_{0}}{d x}\right\rangle=\lambda_{R}^{W}-\lambda_{L}^{W}
$$


2. Due to the CE expansion in the bulk the energy flux $\lambda_{1}$ is of first order in $\varepsilon$. Since it is constant, it is of first order in $\varepsilon$ in the Knudsen layer as well,

$$
\lambda_{1}=\mathcal{O}(\varepsilon) .
$$

3. When written in the Knudsen layer variable $X=x / \varepsilon$, the equations (21) for the Knudsen layer moments $\lambda_{n}(n \geq 2)$ do not contain the Knudsen number. It follows that the amplitudes of all $\lambda_{n}(n \geq 2)$ are of the same order in $\varepsilon$.

4. The boundary conditions (10) for $n \geq 3$ relate the amplitudes of the Knudsen layer moments to the energy jump, so that

$$
\lambda_{n}=\mathcal{O}\left(\bar{\lambda}_{0}-\lambda_{W}\right) \text { for } n \geq 2 .
$$

5. The boundary condition (10) for energy flux reads

$$
\bar{\lambda}_{1}=-\gamma \frac{2 \chi}{2-\chi}\left[\frac{1}{2}\left(\bar{\lambda}_{0}-\lambda_{W}\right)+\sum_{m=2, m \text { even }} \Psi_{1 m} \bar{\lambda}_{m}\right] .
$$

With Point 2 the left hand side is $\mathcal{O}(\varepsilon)$, and with Point 4 the right hand side is $\mathcal{O}\left(\bar{\lambda}_{0}-\lambda_{W}\right)$; both must be equal. Thus, the energy jump can be estimated as

$$
\bar{\lambda}_{0}-\lambda_{W}=\mathcal{O}(\varepsilon) .
$$

6. With Points 4 and 5, the amplitudes of the Knudsen layer moments are of first order in $\varepsilon$,

$$
\lambda_{n}=\mathcal{O}(\varepsilon) \quad \text { for } n \geq 2 .
$$

We emphasize that the CE expansion estimates $\lambda_{2}$ as a second order quantity, see (16), which is true in the bulk only. The present estimate for $\lambda_{2}$ as a first order quantity results from the evaluation of boundary conditions and the bulk equations.

\subsection{Asymptotic reduction}

Now we ask for the results to first order in $\varepsilon$. The result for energy (20) reads

$$
\lambda_{0}=K-\frac{3}{\varepsilon} \lambda_{1} x-2 \lambda_{2}
$$

where $K$ and $\lambda_{1}$ are constants of integration, and $\lambda_{2}$ is the Knudsen layer contribution. The above estimates showed that $\lambda_{1}$ and $\lambda_{2}$ are both of first order in $\varepsilon$. However, since the energy flux $\lambda_{1}$ carries the factor $3 / \varepsilon$, it contributes the leading term, while the influence of the Knudsen layer contribution, $-2 \lambda_{2}$, is one order of magnitude smaller. It follows that the Knudsen layer contribution can be ignored within a first order theory. Accordingly, the expression for energy in the limit of small $\varepsilon$ reduces to the well-known linear law

$$
\lambda_{0}=K-\frac{3}{\varepsilon} \lambda_{1} x .
$$


Boundary conditions (10) for $\lambda_{1}$ are required at both boundaries, they read

$$
\bar{\lambda}_{0}-\lambda_{W}=-\left[\gamma \frac{2-\chi}{\chi} \bar{\lambda}_{1}+2 \sum_{m=2, m \text { even }} \Psi_{1 m} \bar{\lambda}_{m}\right] .
$$

The term in brackets is of first order in $\varepsilon$. For very small $\varepsilon$ it is sufficient to consider only the leading term. Then, the jump correction can be ignored, and energy becomes - approximately - continuous at the boundary,

$$
\bar{\lambda}_{0}=\lambda_{W} \quad(\varepsilon \ll 1) .
$$

This is the classical no-jump boundary condition, which is justified here by an argument based on the smallness of the Knudsen number.

The first order jump (25) contains contributions from higher moments (which can make up for more than $10 \%$ of the jump). On the other hand, the first order equation for energy (24) does not require Knudsen layer contributions for the bulk. Thus, in the first order theory the Knudsen layers appear to be so small that they only contribute directly at the wall, i.e., to the jump.

The usual method to treat Knudsen layer contributions to the jump is to assume that they are proportional to the jump [30]. This gives the jump conditions as

$$
\bar{\lambda}_{0}-\lambda_{W}=-\zeta \gamma \frac{2-\chi}{\chi} \lambda_{1},
$$

with the Knudsen layer correction factor

$$
\zeta=\left[1+\frac{2 \sum_{m=2, m \text { even }} \Psi_{1 m} \bar{\lambda}_{m}}{\left(\bar{\lambda}_{0}-\lambda_{W}\right)}\right]^{-1} .
$$

\section{Results}

\section{$6.1 \varepsilon=0.1$}

First we consider the case of a small Knudsen number, $\varepsilon=0.1$. Figure 1 shows the energy curves for $N=1,3,21$, the differences between the curves are hardly visible in the plot. Only close to the boundary the end of the curve for $N=1$ (light grey) appears from under the curve for $N=3$ (black) which covers the curve for $N=21$ (dark grey) almost completely.

$\varepsilon=0.1$ is small enough to expect validity of the first order theory discussed above. Indeed, the energy curve is essentially linear (24), and there is a jump at the boundary (27). The Knudsen layer contributions are invisible, due to their small amplitudes. Note that the results for $N=1$ do not include the Knudsen layer correction; results with the correction are discussed in Section 6.5.

For smaller $N$ the jump at the wall is slightly overestimated, the values are $\bar{\lambda}_{0}=\{0.882,0.894,0.898\}$ for $N=\{1,3,21\}$ which corresponds to an error of $1.8 \%$ for $N=1$ and $0.5 \%$ for $N=3$. The heat flux assumes the values $\lambda_{1}=\{-0.0588,-0.0584,-0.0584\}$, i.e., the error in heat flux is even smaller. Already at this moderately small Knudsen number, the classical theory with $N=1$ is fairly good.

The eigenvalues and their amplitudes for $N=3,5,21$ are shown in Fig. 2 (left). Only larger eigenvalues, corresponding to wider layers, have relevant 


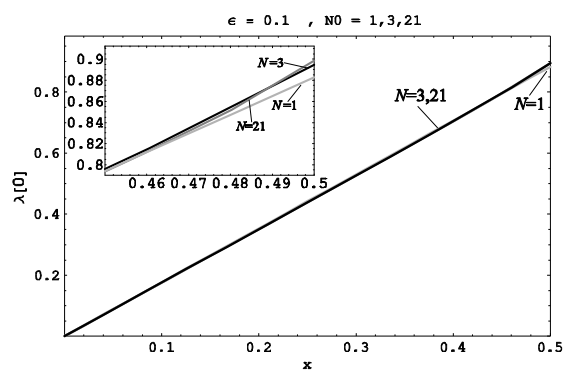

Figure 1: $\varepsilon=0.1$ : Energy density $\lambda_{0}$ computed with $N=1$ (light grey), $N=3$ (black), $N=21$ (dark grey, hidden behind black curve). The prescribed boundary value at $x=0.5$ is $\lambda^{W}=1$. The insert shows a blow-up of the region close to the boundary.
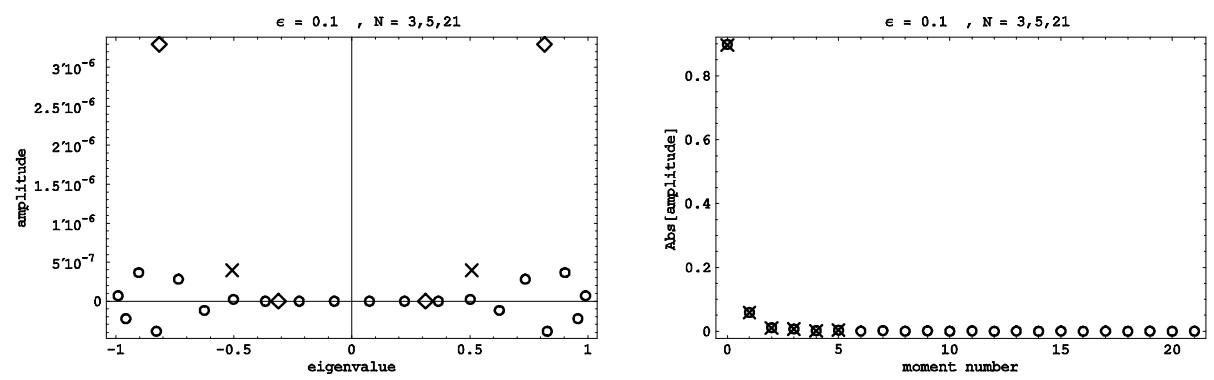

Figure 2: $\varepsilon=0.1$ : LEFT: Eigenvalues $b^{(\alpha)}$ and their amplitudes $\Gamma_{\alpha}^{0}$ for $N=3$ (crosses), $N=5$ (diamonds), and $N=21$ (circles). RIGHT: Absolute values of the moments at the boundary, $\left|\lambda_{n}(0.5)\right|$ for $N=3$ (crosses) and $N=21$ (circles).

amplitudes. For $N=3$ there is only one eigenvalue $( \pm 0.5071)$, which is slightly smaller than the relevant eigenvalues for $N=21$. For $N=5$ we have two eigenvalues, $( \pm 0.8162, \pm 0.3122)$, but only the larger eigenvalue is relevant.

The right part of Fig. 2 shows the fast convergence of the amplitudes of the moments $\lambda_{n}$. Only energy $\lambda_{0}$ and energy flux $\lambda_{1}$ have significant values, while the Knudsen layer moments $\lambda_{n}(n \geq 2)$ are almost zero.

\section{$6.2 \varepsilon=1$}

Next we consider the Knudsen number $\varepsilon=1$, which allows good insight into the overall solution behavior. We observe good convergence of results as $N$ grows, and in the figures we use the results for $N=21$ as reference case, since further increase of $N$ shows no visible changes. Figure 3 (left) shows the energy density for $N=1,3,21$ in the right half of the domain.

We observe a marked difference between the exact result (dark grey), and the classical case $(N=1$, light grey) which does not contain a Knudsen layer and overpredicts the energy jump at the boundary $\left(\lambda_{R}^{W}=1 !\right)$.

For $N=3$ we see a marked improvement: Most of the energy curve is met quite well, with only small differences close to the boundary. The differences 

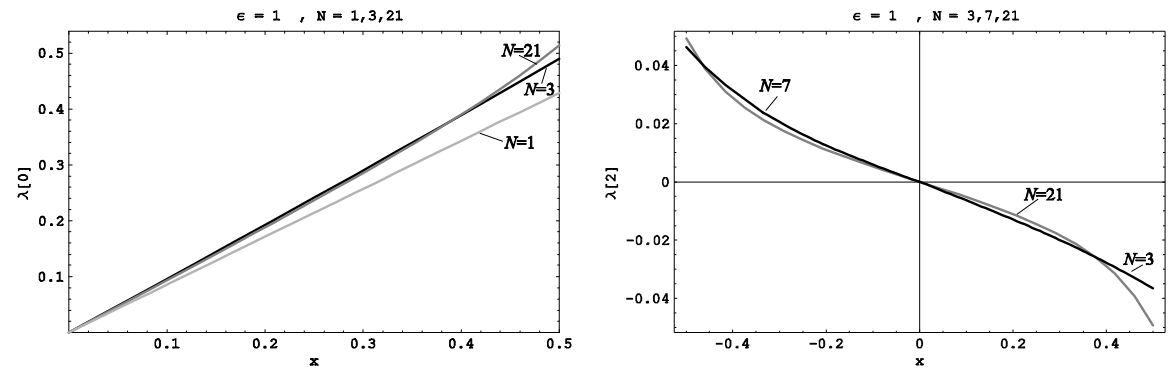

Figure 3: $\varepsilon=1$ : LEFT: Energy density $\lambda_{0}$ computed with $N=1$ (light grey), $N=3$ (black), $N=21$ (darkgrey). The prescribed boundary value at $x=0.5$ is $\lambda^{W}=1$. RIGHT: Second moment $\lambda_{2}$ computed with $N=3$ (black, right half), $N=7$ (black, left half), $N=21$ (darkgrey).
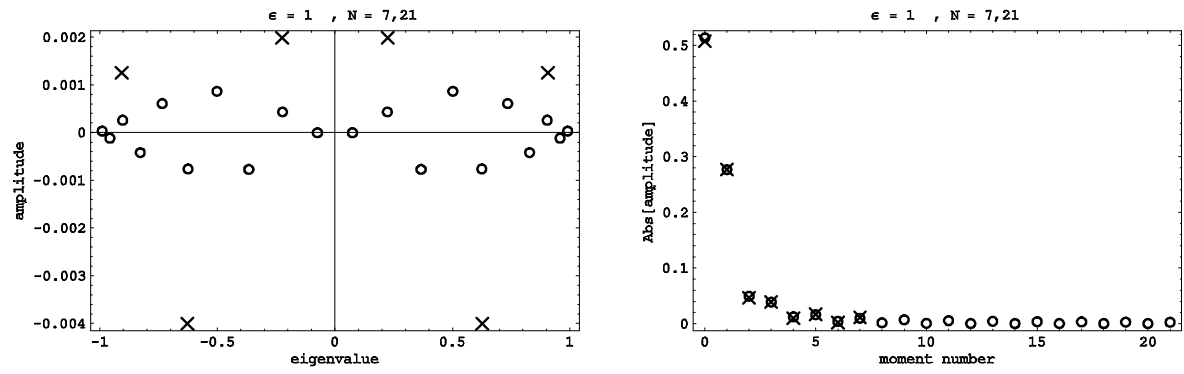

Figure 4: $\varepsilon=1$ : LEFT: Eigenvalues $b^{(\alpha)}$ and their amplitudes $\Gamma_{\alpha}^{0}$ for $N=7$ (crosses) and $N=21$ (circles). RIGHT: Absolute values of the moments at the boundary, $\left|\lambda_{n}(0.5)\right|$ for $N=7$ and $N=21$.

are due to the fact that the proper result is a superposition of Knudsen layers with a variety of widths while for $N=3$ the only eigenvalues are $\{ \pm 0.5071\}$, so that there is only one wider Knudsen layer.

For the energy flux we obtain for $N=\{1,3,7,21\}: \lambda_{1}=\{-0.2857,-0.2779$, $0.2770,-0.2767\}$. Thus, the classical theory $(N=1)$ gives a deviation of $3.2 \%$ compared to the converged result, while the deviation for the first theory with Knudsen layers $(N=3)$ is only $0.4 \%$.

Figure 3 (right) shows the second moment $\lambda_{2}$ (which describes the deviation from the Fourier solution, see (20)) for $N=3,7,21$, where the left part of the figure shows the solution for $N=7$, and the right part that for $N=3$. The case $N=7$ has the eigenvalues $\{ \pm 0.9065, \pm 0.6282, \pm 0.2243\}$ and thus can describe the structure of the actual Knudsen layer in greater detail.

Figure 4 shows on the left, for $N=7$ and $N=21$, the eigenvalues and their respective amplitudes. All eigenvalues contribute to the Knudsen layers with different amplitudes, with larger amplitudes for eigenvalues around 0.5. For $N=7$ the amplitudes are larger compared to those for $N=21$, since fewer eigenvalues contribute. The superposition of the Knudsen layers leads to the excellent agreement reported in Fig. 3

The right Fig. 4 shows, again for $N=7$ and $N=21$, the absolute values of 


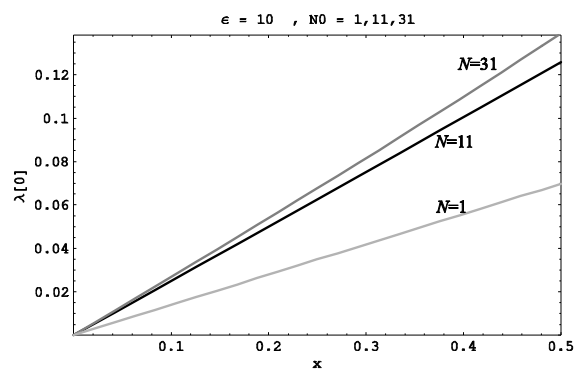

Figure 5: $\varepsilon=10$ : Energy density $\lambda_{0}$ computed with $N=1$ (light grey), $N=11$ (black), $N=31$ (darkgrey). The prescribed boundary value at $x=0.5$ is $\lambda^{W}=1$.
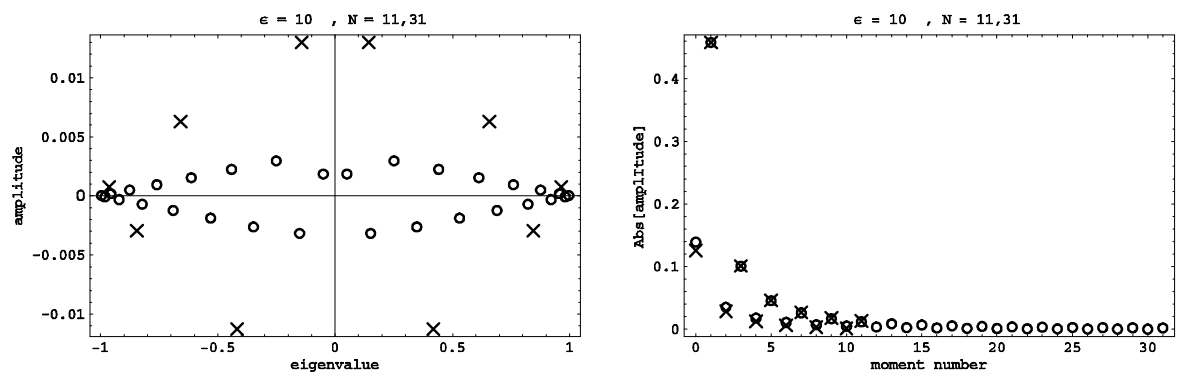

Figure 6: $\varepsilon=10$ : LEFT: Eigenvalues $b^{(l)}$ and their amplitudes $\Gamma_{\alpha}^{0}$ for $N=11$ (crosses) and $N=31$ (circles). RIGHT: Absolute values of moments at boundary, $\left|\lambda_{n}(0.5)\right|$ for $N=11,31$.

the moments at the right boundary. We observe excellent agreement between the results for all moments for different $N$.

In addition, the figure shows that the amplitudes of the higher moments are considerably smaller than those for the lower moments. This decrease in amplitude with growing $n$ cannot be attributed to the smallness parameter ( $\varepsilon=1$ and thus is not small!), but to the geometry and the properties of the equation system.

\section{$6.3 \varepsilon=10$}

For larger $\varepsilon$ the behavior is similar, but convergence is slower. We present some results for $\varepsilon=10$, where results are converged for $N=31$.

Figure 5 shows the energy density for with $N=1,11,31$, and exhibits marked energy jumps at the boundary (recall that the boundary value is $\lambda_{W}=1$ ), and pronounced differences between the results for different $N$.

The eigenvalues and their corresponding amplitudes are shown in Fig. 6 (left), for $N=11,31$. All eigenvalues contribute; compared to the case $\varepsilon=1$ smaller eigenvalues become more important.

The values of the moments $\lambda_{n}$ at the wall are depicted in Fig. 6 (right). As for $\varepsilon=1$, we observe a decrease of moment amplitude with moment number, but now the decrease is slower, reflecting the slower convergence with $N$. 

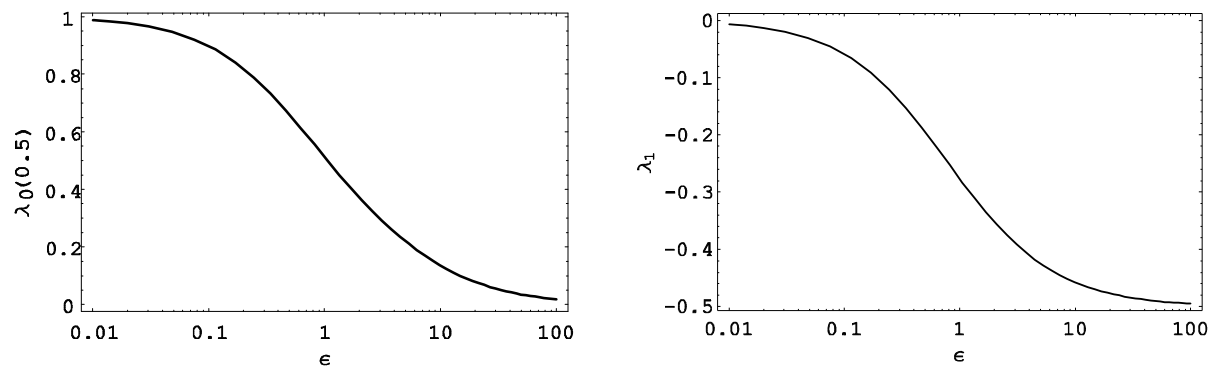

Figure 7: Energy $\lambda_{0}$ at the boundary (left) and energy flux $\lambda_{1}$ (right) over Knudsen number $\varepsilon$ for $N=31$.

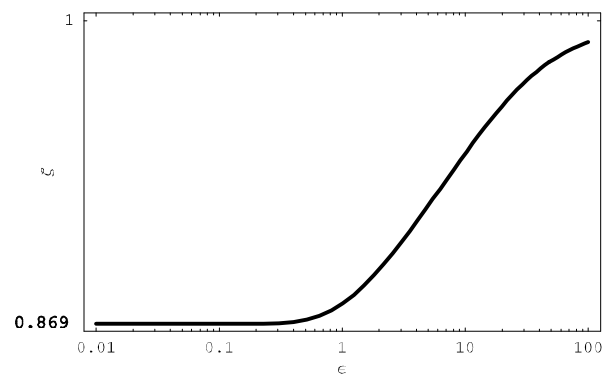

Figure 8: Knudsen layer correction coefficient $\zeta$ (28) over Knudsen number; both axes are logarithmic.

\section{$6.40<\varepsilon<\infty$}

We study the behavior of the first two moments with the Knudsen number $\varepsilon$, for $N=31$.

Figure 7 (left) shows the energy at the right wall. For small $\varepsilon$ the value $\lambda_{0}(0.5)$ agrees with the prescribed value $\lambda_{W}=1$. As $\varepsilon$ grows the energy jump grows as well, and becomes maximal as $\varepsilon \rightarrow \infty$, that is for free molecular flow. In this case the gas is a mixture of particles moving in free flight between the two boundaries, which carry the energies $\lambda_{L, R}^{W}$ of the respective wall; the average energy is $\frac{1}{2}\left(\lambda_{W}^{R}+\lambda_{W}^{L}\right)=0$.

The curve for the energy flux looks very similar, see Fig. 7 (right). As the Knudsen number grows, more and more heat is transported, up to the maximum value for free molecular flow, $\lambda_{1}^{\max }=-0.5$.

\subsection{Knudsen layer correction}

Figure 8 shows the correction coefficient (28) for $\varepsilon \in(0.01,100), \chi=1, N=31$. For Knudsen numbers $\varepsilon \leq 0.3$ the correction coefficient assumes the constant value $\zeta=0.869$, which should be used in (27). The value of $\zeta$ depends on the accommodation coefficient $\chi$; our computations suggest the linear relationship $\zeta=1-0.131 \chi$ 

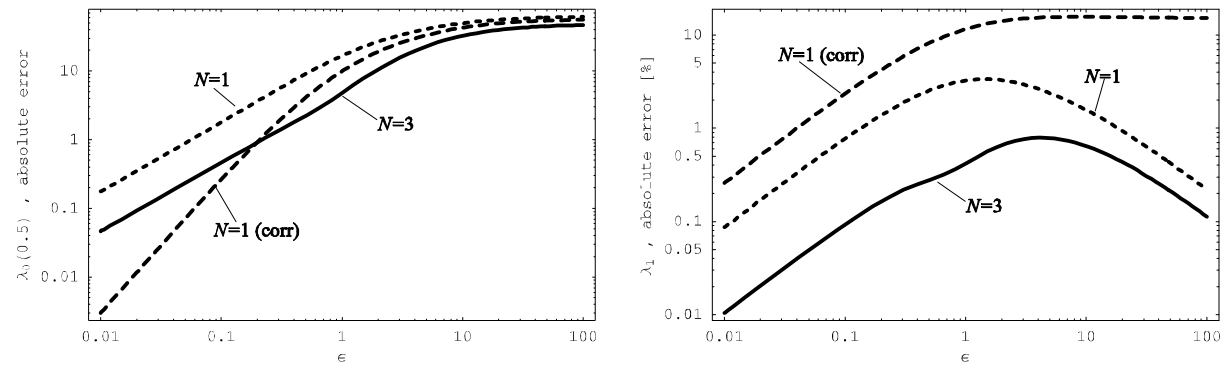

Figure 9: Deviation (in \%) of energy $\lambda_{0}$ at boundary (left) and energy flux $\lambda_{1}$ (right) from the converged case $(N=31)$, for $N=3$ (continuous), $N=1$ (long dashes), and $N=1$ with Knudsen layer correction $\zeta=0.869$ (short dashes)

For $\zeta=0.869, \chi=1$, Fig. 9 shows on the left the relative error for the boundary value of $\lambda_{0}$ compared to the converged solution $(N=31)$. In addition, the relative error for the the case $N=3$ is given, which is the simplest case with a Knudsen layer. The correction (27) allows to achieve very small deviations for smaller Knudsen numbers $\varepsilon$. This improvement for the energy jump is, however, accompanied by an increase in the deviation of the energy flux, as becomes clear from the right figure. Thus, the Knudsen layer correction improves the accuracy of the energy curves, but reduces the accuracy of the energy flux.

For $N=1$ the error becomes larger than $5 \%$ for $\varepsilon \simeq 0.3$ with and without the Knudsen layer correction. For the uncorrected case, $\zeta=1$, the deviation of boundary energy exceeds the error limit, while for the corrected case, $\zeta=0.846$, the energy flux exceeds the limit.

For $N=3$, however, the error limit of $5 \%$ is exceeded only for $\varepsilon>1$. The energy curve for this case was shown in Fig. 3, and deviates only in a small region around the boundary from the exact results.

The results in this section suggest that is more beneficial to use a moment system that at least has one pair of Knudsen layers, than considering the classical limit with jump conditions and Knudsen layer correction.

\subsection{Moment number and accuracy}

In order to discuss the relation between Knudsen number, number of moments, and accuracy, we study the distribution function. For the collisionless case $(\varepsilon \rightarrow \infty)$ the distribution is determined only through the boundary conditions (5), and it is independent of the space variable, and thus discontinuous in $\mu$ everywhere in the domain,

$$
f_{\varepsilon \rightarrow \infty}=\left\{\begin{array}{ll}
\frac{1}{2(2-\chi)}\left[\lambda_{L}^{W}+(1-\chi) \lambda_{R}^{W}\right] & , \quad \mu>0 \\
\frac{1}{2(2-\chi)}\left[\lambda_{R}^{W}+(1-\chi) \lambda_{L}^{W}\right] & , \quad \mu<0
\end{array} .\right.
$$

A large number $N$ of polynomials is required to have a good approximation of the discontinuous phase density $f_{\varepsilon \rightarrow \infty}$ through Legendre polynomials (6) everywhere in $\left(-\frac{1}{2}, \frac{1}{2}\right)$.

When $\varepsilon$ is finite, collisions occur that smooth the phase density further away from the wall, while discontinuities are observed only close to the walls. This 


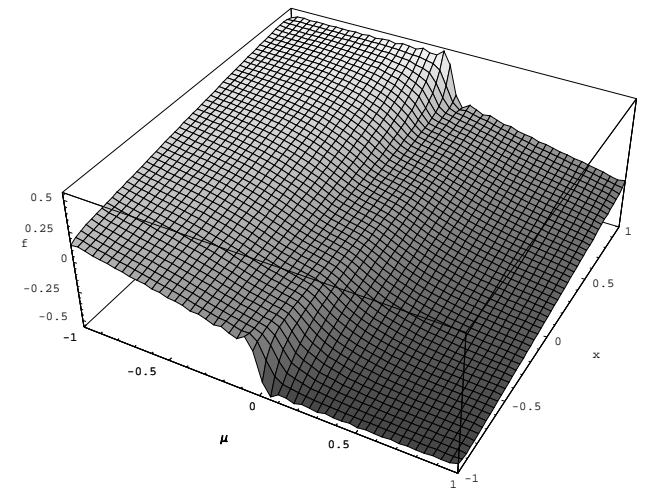

Figure 10: Distribution function $f(x, \mu)$ for $\varepsilon=2$, with $N=51, \chi=1, \lambda_{W}^{L}=$ $-\lambda_{W}^{R}=1$.

behavior is depicted in Fig. 10 which shows the distribution function for $\varepsilon=$ 2. A smooth function can be approximated reasonably well by few Legendre polynomials, and thus a low number of moments will allow a good description of the distribution function at those locations where the distribution is smooth.

Thus, the overall error will be small when the distribution function is smooth in most of the domain. Discontinuities adjacent to the walls will be poorly described by a theory with only few moments, but will, as long as the region of their existence is small compared to the whole domain, contribute only little to the overall error (think of the space integral of the error). Thus, few moments suffice when the distribution function is smooth in most of the domain.

\section{Conclusions and conjectures}

We discussed Knudsen layers for a simple one-dimensional, linear kinetic equation. From the asymptotics for the Knudsen layers follows that these can be ignored only in a theory of first order in the Knudsen number, which corresponds to classical transport theory (Fourier's law, in our case).

Knudsen layers must be included, however, for theories of second order or higher (Burnett level, and higher in the language of the CE method). The Knudsen layers arise from an eigenvalue problem for the moment equations, and thus involve all moment equations equally. Therefore, there is no a priori argument to limit the number of moments that must be considered for an accurate description. The moments are superpositions of Knudsen layer contributions of different widths, the results become more accurate when a greater variety of widths is available. Fortunately, for a given number $N$ of moments, the eigenvalues are almost evenly distributed, so that already for relatively small $N$ good results can be obtained. Indeed, the moment systems appear to be best approximations [31].

As long as discontinuities of the distribution function - which are inevitably induced at the boundaries - are present only in a relatively small part of the space domain, it is sufficient to consider few Knudsen layer contributions. In the present application, a set of moment equations with few moments $(N=3)$ 
can give satisfactory results for Knudsen numbers $\varepsilon \leq 1$.

We close with some conjectures concerning moment equations for the full Boltzmann equation, which are more complicated, since the gas particles are described microscopically not only by their direction, but also by the magnitude of their velocities. Nevertheless, the following statements can be transferred to the moment system of the Boltzmann equation:

- Knudsen layers are a second order effect, and thus will not appear in hydromechanical models, but only in extensions to include higher order effects in the Knudsen number.

- A proper moment set should allow for the description of Knudsen layers for all variables.

- A larger number of moments allows finer resolution of Knudsen layers.

- Boundary conditions for extended moment sets can be derived from the boundary conditions for the phase density.

Within the theory of moment equations for the full Boltzmann equation, the Burnett and super-Burnett equations have only some Knudsen layers, plus some unphysical (oscillatory) contributions, and Grad's 13 moment equations display no Knudsen layers at all [11]. The first moment system with a full set of Knudsen layers are the R13 equations which can be derived from the 26 moment case [18][11]. The 26 moment case [32] has additional Knudsen layers for the higher moments. Thus, for linear problems, where Knudsen layer effects are particularly important, one will need to consider at least the R13 equations (for which a theory for boundary conditions is now available $[22,23]$ ).

Strongly non-linear processes are dominated by bulk effects, and for these the Chapman-Enskog ordering can be applied. Then, Grad's 13 moment equations are appropriate to second order in the Knudsen number [21][33], and the R13 equations are appropriate to third order [20].

Acknowledgments: Support by the Natural Sciences and Engineering Research Council (NSERC) is gratefully acknowledged. The paper was written during my stay as Gastprofessor at ETH Zürich, and I like to thank Prof. Hans Christian Öttinger and his group at the Institute for Polymer Physics for their kind hospitality. Also I thank M. Torrilhon (ETH Zürich) for stimulating discussions.

\section{References}

[1] G. E. Karniadakis, A. Beskok, and N. Aluru, Microflows and Nanoflows: Fundamentals and Simulation. Springer, New York 2005

[2] C. Cercignani, Slow Rarefied Flows. Theory and Application to MicroElectro-Mechanical Systems. Birkhäuser, Basel 2006

[3] S. Chapman and T. G. Cowling, The Mathematical Theory of Non-Uniform Gases. Cambridge University Press 1970

[4] M.N. Kogan, Rarefied Gas Dynamics. Plenum Press, New York 1969 
[5] P.A. Markowich, C.A. Ringhofer, and C. Schmeiser. Semiconductor equations. Springer, Wien 1990

[6] W. Dreyer and H. Struchtrup, Heat Pulse Experiments Revisited, Cont. Mech. Thermodyn. 5 (1993), 3-50

[7] S. Chandrasekhar, Radiative Transfer. Dover Pub., London (1960)

[8] H. Struchtrup, Macroscopic Transport Equations for Rarefied Gas FlowsApproximation Methods in Kinetic Theory. Interaction of Mechanics and Mathematics Series, Springer, Heidelberg 2005

[9] A.V. Bobylev, The Chapman-Enskog and Grad methods for solving the Boltzmann equation, Sov. Phys. Dokl. (1982) 27, 29-31

[10] P. Rosenau, Extending hydrodynamics via the regularization of the Chapman-Enskog solution, Phys. Rev. A 40 (1989), 7193-7196

[11] H. Struchtrup, Failures of the Burnett and Super-Burnett equations in steady state processes, Cont. Mech. Thermodyn. 17 (2005), 43-50

[12] H. Grad, On the Kinetic Theory of Rarefied Gases, Comm. Pure Appl. Math. 2 (1949), 331-407

[13] H. Grad, Principles of the Kinetic Theory of Gases, in Handbuch der Physik XII: Thermodynamik der Gase, S. Flügge (Ed.), Springer, Berlin 1958

[14] I. Müller and T. Ruggeri, Rational Extended Thermodynamics. Springer, New York 1998 (Springer Tracts in Natural Philosophy Vol. 37)

[15] C. D. Levermore, Moment closure hierarchies for kinetic theories, J. Stat. Phys. 83 (1996), 1021-1065

[16] H. Struchtrup, Heat Transfer in the Transition Regime: Solution of Boundary Value Problems for Grad's Moment Equations via Kinetic Schemes, Phys. Rev. E 65 (2002), 041204

[17] H. Struchtrup, Grad's Moment Equations for Microscale Flows, in 23rd Intl. Symposium on Rarefied Gas Dynamics, AIP Proceedings 663 (2003), 792-799

[18] H. Struchtrup and M. Torrilhon, Regularization of Grad's 13-momentequations: Derivation and Linear Analysis, Phys. Fluids 15 (2003), 26682680

[19] M. Torrilhon and H. Struchtrup, Regularized 13-Moment-Equations: Shock Structure Calculations and Comparison to Burnett Models, J. Fluid Mech. 513 (2004), 171-198

[20] H. Struchtrup, Stable transport equations for rarefied gases at high orders in the Knudsen number, Phys. Fluids 16 (2004), 3921-3934

[21] H. Struchtrup and T. Thatcher, Bulk equations and Knudsen layers for the regularized 13 moment equations, Cont. Mech. Thermodyn. 19 (2007), $177-189$ 
[22] X. Gu and D. Emerson, A Computational Strategy for the Regularized 13 Moment Equations with Enhanced Wall-Boundary Conditions, J. Comp. Phys. 225 (2007), 263-283

[23] M. Torrilhon and H. Struchtrup, Boundary Conditions for Regularized 13Moment-Equations for Micro-Channel-Flows, J. Comp. Phys., published online 17 October 2007, doi:10.1016/j.jcp.2007.10.006

[24] A.M. Anile, V. Romano, and G. Russo, Extended hydrodynamical model of carrier transport in semiconductors, SIAM J. Appl. Math. 61 (2000), 76-101

[25] H. Struchtrup, Extended moment method for electrons in semiconductors, Physica A 275 (2000), 229-255

[26] H. Struchtrup, On the number of moments in Radiative transfer problems, Ann. Phys. 266 (1998), 1-26

[27] Y. Sone, Kinetic Theory and Fluid dynamics. Birkhäuser, Boston 2002

[28] J. C. Maxwell, On Stresses in Rarefied Gases Arising From Inequalities of Temperature, Phil. Trans. Roy. Soc. (London) 170 (1879), 231-256

[29] G.-W. Yuan, Convergence of PN approximation for the neutron transport equation with reflective boundary condition, J. Math. Phys. 41 (2000), 867874

[30] F. Sharipov and V. Seleznev, Data on Internal Rarefied Gas Flows, J. Phys.Chem. Ref. Data 27 (1998), 657-706

[31] F. Deutsch, Best Approximations in Inner Product Spaces, Springer, NewYork 2001

[32] D. Reitebuch and W. Weiss, Application of high moment theory to the plane Couette flow, Cont. Mech. Thermodyn. 11 (1999), 217-225

[33] W. Marques Jr. and G.M. Kremer, Couette flow from a thirteen field theory with jump and slip boundary conditions. Continuum Mech. Thermodyn. 13 (2001), 207-217 